\title{
LISTA RECENZENTÓW „RUCHU PRAWNICZEGO, EKONOMICZNEGO I SOCJOLOGICZNEGO” ZA ROK 2018
}

Katarzyna Andrejuk

Marek Andrzejewski

Andrzej Bator

Teresa Bednarczyk

Izabela Bludnik

Marcin Byczyk

Ewelina Cała-Wacinkiewicz

Teresa Chirkowska-Smolak

Maciej Cieślukowski

Wojciech Dajczak

Marek Dobrowolski

Rafał Drozdowski

Tomasz Duraj

Aniela Dylus

Joanna Dzionek-Kozłowska

Ewa Frąckiewicz

Tadeusz Gadkowski

Dominik Gajewski

Anna Gerecka-Żołyńska

Grzegorz Gołębiowski

Justyna Goździewicz-Biechońska

Sabina Grabowska

Radosław Grabowski

Beata Guziejewska

Joanna Haberko

Piotr Jabkowski

Barbara Janusz-Pohl

Andrzej Jarocha

Andrzej Jędruchniewicz

Sławomir Kalinowski

Sławomira Kańduła

Leszek Karczewski

Karol Kiczka

Katarzyna Klafkowska-Waśniowska

Tadeusz Kocowski

Eryk Kosiński

Olga Kowalczyk

Michał Krotoszyński

Łukasz Kułaga

Katarzyna Kuziak

Irena Lipowicz

Piotr Lisowski

Wojciech Łączkowski

Edyta Małecka-Ziembińska

Kamilla Marchewka-Bartkowiak

Paweł Marszałek

Małgorzata Masternak-Kubiak

Dominik Mączyński
Danuta Miłaszewicz

Anna Młynarska-Sobaczewska

Jacek Napierała

Krystyna Nizioł

Aleksandra Nowakowska

Aurelia Nowicka

Marcin Olszak

Jerzy Olszewski

Wojciech Orłowski

Wojciech Patryas

Wojciech Piątek

Ryszard Piotrowski

Łukasz Pohl

Marzanna Poniatowicz

Bożena Popowska

Mariusz Próchniak

Wiesława Przybylska-Kapuścińska

Anna Rakowska

Marek Ratajczak

Wanda Ronka-Chmielowiec

Antoni Rost

Mirosław Sadowski

Przemysław Sadura

Jan Sandorski

Rafał Sikorski

Michał Skapski

Jędrzej Skrzypczak

Marek Smolak

Wojciech Staszewski

Mirosław Stec

Magdalena Stefańska

Jerzy Stelmach

Hanna Suchocka

Wojciech Szafrański

Marek Szewczyk

Krzysztof Ślebzak

Maria Trojanek

Artur A. Trzebiński

Marcin Walasik

Marzenna Weresa

Paweł Wiliński

Zbigniew Woźniak

Jerzy Zajadło

Robert Zawłocki

Anna Zielińska-Głębocka

Krystian Ziemski

Katarzyna Żukrowska

Aldona Żurek 